Ambiente \& Água - An Interdisciplinary Journal of Applied Science
ISSN 1980-993X - doi:10.4136/1980-993X
www.ambi-agua.net
E-mail: ambi.agua@gmail.com

\title{
Efeitos de poluentes do ar e doenças respiratórias utilizando dados estimados por modelo matemático
}

\author{
doi:10.4136/ambi-agua.1592 \\ Received: 13 Jan. 2015; Accepted: 10 Aug. 2015 \\ Vanessa Villalta Lima Roman ${ }^{1}$; João Andrade de Carvalho Jr1 ; \\ Luiz Fernando Nascimento ${ }^{2 *}$; Ana Cristina G. Cesar ${ }^{3}$ \\ ${ }^{1}$ Universidade Estadual Paulista “Júlio de Mesquita Filho" (UNESP), Guaratinguetá, SP, Brasil \\ Departamento de Energia \\ ${ }^{2}$ Universidade de Taubaté (UNITAU), Taubaté, SP, Brasil \\ Departamento de Medicina \\ ${ }^{3}$ Instituto Federal de Educação, Ciência e Tecnologia de São Paulo, Bragança Paulista, SP, Brasil \\ *Autor correspondente: e-mail: luiz.nascimento@unitau.com.br, \\ vanessa@proecologic.com.br, joao@feg.unesp.br, anagobbo@ifsp.edu.br
}

\section{RESUMO}

Este artigo utilizou concentrações de poluentes do ar estimados por modelo matemático para identificar associações com doenças respiratórias. Os dados de internação de residentes em Taubaté, SP, por doenças respiratórias (CID-10 J00 a J99), foram obtidos do Datasus no período de 01.08.2011 a 31.07.2012. Os valores das concentrações estimadas dos poluentes $\left(\mathrm{CO}, \mathrm{PM}_{2.5}, \mathrm{O}_{3}, \mathrm{NO}_{\mathrm{x}}\right)$ foram fornecidas pelo modelo matemático CATT-BRAMS (CPTEC-INPE). Os dados diários das variáveis meteorológicas (temperatura e umidade relativa) foram obtidas no CPTEC-INPE e usadas para cálculo da temperatura aparente, usada para ajustar o modelo multipoluente. Para a análise utilizou-se o modelo aditivo generalizado de regressão de Poisson, com estrutura de defasagem de zero a cinco dias. Foram 352 internações no período. A exposição a óxidos de nitrogênio na defasagem de um dia apresentou $\mathrm{RR}=1,046$ (IC 95\%: 1,015-1,079) e na defasagem de quatro dias, $\mathrm{RR}=1,054$ (IC 95\%: 1,054-1,196). O incremento na concentração de $3 \mu \mathrm{g} / \mathrm{m}^{3}$ deste poluente implicou na elevação em até $17 \%$ no risco de internação. A utilização de dados estimados por modelo matemático identificou associação entre exposição aos poluentes do ar e as internações por doenças respiratórias, permitindo a utilização deste modelo em cidades onde não existam estações medidoras de poluentes do ar.

Palavras-chave: dióxido de nitrogênio, modelagem, poluição do ar.

\section{Effects of air pollutants on respiratory diseases using estimated values by mathematical modelling of simulation}

\section{ABSTRACT}

This study used a mathematical model to associate estimated concentrations of air pollutants with respiratory diseases. Admission data were obtained from Datasus relating to the hospitalization of residents in Taubaté due to respiratory diseases between 08/01/2011 and 07/31/2012. Values of the estimated air pollutant concentrations $\left(\mathrm{CO}, \mathrm{PM}_{2.5}, \mathrm{O}_{3}, \mathrm{NO}_{\mathrm{x}}\right)$ were 
provided by the CATT-BRAMS (CPTEC-INPE) mathematical model. Daily data regarding temperature and relative humidity were obtained from CPTEC-INPE and used to calculate the apparent temperature, and then used to adjust the multipollutant model. The analysis used the generalized additive model Poisson regression, and lags of zero to five days. Three hundred and fifty two admissions occurred during the period analyzed. Exposures to oxides of nitrogen in the one day lag showed $\mathrm{RR}=1.046$ (95\% CI: 1.015-1.079) and at four days lag showed a $\mathrm{RR}=1.054$ (95\% CI: $1.054-1.196)$. The risk of hospitalization increases up to $17 \%$ when the concentrations of oxides of nitrogen increase by the amount of $3 \mu \mathrm{g} / \mathrm{m}^{3}$. The use of estimated data by mathematical model identified an association between exposure to air pollutants and hospitalization due to respiratory diseases, validating the use of this model in cities where there are no air pollutant measuring stations.

Keywords: air pollution, nitrogen dioxide, mathematical model.

\section{INTRODUÇÃO}

No ano de 2012, as doenças respiratórias (DR) foram responsáveis por mais de 1,3 milhão de internações no Brasil, sendo que em São Paulo foram cerca de 240 mil, gerando custos da ordem de R\$ 1, 3 bilhão e em São Paulo, R\$ 240 milhões (Brasil, 2014a) $(1 \mathrm{US} \$ \approx \mathrm{R} \$ 2,00)$. Neste mesmo ano foram relatadas 125 mil mortes por doenças respiratórias, 34 mil em São Paulo (Brasil, 2014b).

Os poluentes mais comumente estudados e associados às DRs são material particulado $(\mathrm{PM})$, ozônio $\left(\mathrm{O}_{3}\right)$, dióxido de enxofre $\left(\mathrm{SO}_{2}\right)$, óxidos de nitrogênio (NOx) e monóxido de carbono (CO) (Arbex et al., 2012). Dentre alguns estudos mostrando os efeitos destes poluentes, podem ser destacados aqueles que apontaram os efeitos do $\mathrm{PM}_{10}$ e $\mathrm{SO}_{2}$ nas internações por asma em crianças (Amâncio e Nascimento, 2012), internações por pneumonias e $\mathrm{PM}_{10}$ em crianças em Cubatão (Jasinski et al., 2011), exposição a $\mathrm{PM}_{10}$, CO, $\mathrm{NO}_{2}, \mathrm{O}_{3}$ e $\mathrm{SO}_{2}$ e internações por DRs em adultos e crianças (Gouveia et al., 2006), $\mathrm{NO}_{2}$ em crianças (Negrisoli e Nascimento, 2013).

Estes poluentes costumam ser quantificados por instrumental de agências ambientais estaduais, no caso do estado de São Paulo, a Companhia de Tecnologia de Saneamento Ambiental (CETESB) é a responsável pela manutenção e coleta diária desses dados. No entanto, mesmo em estados onde há agência ambiental, não são todas as cidades que contam com estes medidores, impossibilitando o monitoramento dos efeitos destes poluentes sobre a saúde da população.

Uma alternativa para os municípios que não apresentam medidores de poluentes é a utilização de dados estimados por modelo matemático como o Coupled Aerosol and Tracer Transport Model to the Brazilian Developments on the Regional Atmospheric Modeling System (CATT-BRAMS) (Freitas et al., 2007). Este sistema que cobre a América do Sul, considera a dinâmica atmosférica e utiliza a emissão e transporte de vários tipos de gases e partículas de aerossol, obtido por satélites, para gerar estimativas diárias de diversos poluentes. O emprego deste modelo matemático foi validado por meio da avaliação dos impactos na saúde humana em decorrência da exposição às emissões de material particulado $\left(\mathrm{PM}_{2.5}\right)$, originado da queima de biomassa na Amazônia brasileira. $\mathrm{O}$ uso do $\mathrm{PM}_{2.5}$ previsto por este modelo numérico foi uma abordagem inovadora e se tornou uma alternativa viável para a rede de monitorização da qualidade do ar tradicional (Ignotti et al., 2010).

Dados estimados por este modelo identificaram os efeitos da exposição ao $\mathrm{PM}_{2.5}$, material com diâmetro aerodinâmico menor que 2,5 micra designado como particulado fino e principal fração do $\mathrm{PM}_{10}$ na saúde humana, sendo que, nestes estudos, o desfecho analisado foram as internações por pneumonias (César et al., 2013; Silva et al., 2013). As principais fontes geradoras deste poluente são a queima de biomassa e de combustíveis fósseis. 
Outro poluente, os óxidos de nitrogênio são compostos por dióxido de nitrogênio $\left(\mathrm{NO}_{2}\right)$, óxido nítrico (NO) e óxido nitroso $\left(\mathrm{N}_{2} \mathrm{O}\right)$ e, gerados por queima de combustíveis fósseis e usinas termoelétricas. A maior parte dos NOx formada está presente como NO, mas esta espécie é rapidamente oxidada a $\mathrm{NO}_{2}$ pela ação do ozônio, de maneira que as taxas de $\mathrm{NOx}$ são comparados com os valores para o $\mathrm{NO}_{2}$ normalizado (Hernandez, 2013).

$\mathrm{O}$ ozônio troposférico $\left(\mathrm{O}_{3}\right)$ é um poluente secundário formado pela ação da radiação ultravioleta sobre os óxidos de nitrogênio, atuando como poderoso agente oxidante. Já o monóxido de carbono $(\mathrm{CO})$ produzido por queimadas florestais, transportes rodoviários, combustão incompleta de combustíveis fósseis ou de outros materiais orgânicos, une-se fortemente com a hemoglobina, interferindo no transporte de oxigênio (Arbex et al., 2012).

Assim, o objetivo deste estudo foi estimar os efeitos da exposição aos poluentes do ar, no município de Taubaté, SP, nas internações por doenças respiratórias, utilizando dados estimados por modelagem matemática.

\section{MATERIAL E MÉTODOS}

Foi desenvolvido um estudo ecológico de série temporal com dados relativos à saúde e de poluentes do ar, no município de Taubaté, SP, no período de 01 de agosto de 2011 a 31 de julho de 2012.

Taubaté situa-se a $23^{\circ} 01^{\prime}$ de latitude sul e $45^{\circ} 33^{\prime}$ de longitude oeste, na mesorregião do Vale do Paraíba, distante $123 \mathrm{~km}$ da capital paulista, $280 \mathrm{~km}$ da cidade do Rio de Janeiro; situa-se entre as serras do Mar e da Mantiqueira e é cortada pela Via Dutra, que apresenta alta densidade de tráfego, em especial de caminhões pesados e ônibus. Seu território abrange pouco mais de $625 \mathrm{~km}^{2}$ e abriga uma população estimada em cerca de 300 mil habitantes (IBGE, 2015). A frota veicular em outubro de 2014 atingiu aproximadamente 193 mil veículos (DENATRAN, 2014).

A variável dependente foi o número de internação por doenças respiratórias obtido no portal do Sistema Único de Saúde - DATASUS e identificados de acordo com o Capítulo X da Classificação Internacional de Doenças, décima revisão (CID- 10) (J00 - J99), por local de residência. As variáveis independentes foram as concentrações dos poluentes $\mathrm{CO}, \mathrm{PM}_{2.5}, \mathrm{O}_{3}$, NOx estimadas pelo modelo CATT-BRAMS. Para ajustar o modelo multipoluente foi utilizada a temperatura aparente, que combina os efeitos do calor e frio, com a umidade (Barnett et al., 2010).

O modelo CATT-BRAMS foi concebido para simular a circulação de poluentes em escala continental e, para tanto, a resolução interna de seus módulos varia de $150 \mathrm{~km}$ a $15 \mathrm{~km}$ cada célula. O município de Taubaté, considerando-se as áreas urbana e rural, mede aproximadamente $17 \times 25 \mathrm{~km}$ e a área urbana, onde se concentra a maior parte da população, mede aproximadamente $8 \times 7 \mathrm{~km}$. Apesar da existência de um conflito de resolução espacial entre o que o modelo é capaz de representar e a área de abrangência da população exposta aos poluentes, estudos empregando os dados estimados gerados pelo CATT-BRAMS têm sido publicados por revista de impacto na literatura internacional, demonstrando a aceitação da aplicação destes dados estimados no monitoramento dos efeitos dos poluentes atmosféricos sobre doenças respiratórias (Ignotti et al., 2010; Silva et al., 2013; César et al., 2013).

As análises foram realizadas usando todos os poluentes simultaneamente (modelo multipoluente), utilizando o Modelo Aditivo Generalizado de Regressão de Poisson, que fornece o risco relativo da ocorrência do desfecho em estudo. Foram calculados os riscos relativos para internações por doenças respiratórias. Como os efeitos da exposição podem ser fazer sentir não só no mesmo dia como em dias posteriores foram utilizadas defasagens de $0 \mathrm{a}$ 5 dias - (lag). Nesta análise foi considerado um incremento correspondente a diferença 
interquartil para o poluente que apresentasse risco relativo significativo, obtendo-se o aumento percentual.

Os aumentos percentuais (AP) dos Riscos Relativos (RR) para as internações, para cada situação foram calculados utilizando Equação 1.

$$
\mathrm{AP}=\left[\exp \left(\beta^{*} \Delta \mathrm{C}\right)-1\right]^{*} 100
$$

Nesta equação $\beta$ é o coeficiente fornecido pela regressão de Poisson e $\Delta C$ é o incremento correspondente à diferença interquartil para o poluente cuja exposição se mostrou associada à internação por DR.

\section{RESULTADOS E DISCUSSÃO}

No período de estudo ocorreram 352 internações por doenças respiratórias, independentes da idade e sexo dos pacientes, residentes no município de Taubaté, SP.

Os valores obtidos para a distribuição de frequência das variáveis independentes monóxido de carbono $(\mathrm{CO})$, ozônio $\left(\mathrm{O}_{3}\right)$, óxidos de nitrogênio ( $\mathrm{NOx}$ ) e dióxido de nitrogênio $\left(\mathrm{NO}_{2}\right)$, material particulado $\left(\mathrm{PM}_{2.5}\right)$, temperatura aparente (TA), e dependentes (casos de internação) expressas com média, desvio-padrão, valores mínimo e máximo do período avaliado são apresentados na Tabela 1.

Os valores das concentrações dos poluentes estiveram altamente correlacionados entre si, sendo que as concentrações de $\mathrm{NOx}$ e $\mathrm{O}_{3}$ se mostraram negativa e significativamente correlacionadas.

Tabela 1. Análise descritiva das variáveis independentes $\left(\mathrm{CO}, \mathrm{O}_{3}, \mathrm{NOx}, \mathrm{PM}_{2.5}, \mathrm{TA}\right)$ e dependentes, Taubaté, SP, $2011-2012$.

\begin{tabular}{lccc}
\hline Variáveis & Média $(\mathbf{D P})$ & Mínimo & Máximo \\
\hline $\mathrm{RESP}^{\#}$ & $0.96(1.08)$ & 0 & 5 \\
$\mathrm{CO}(\mathrm{ppb})$ & $130.04(52.81)$ & 33.3 & 550.0 \\
$\mathrm{O}_{3}\left(\mu \mathrm{g} / \mathrm{m}^{3}\right)$ & $61.98(18.47)$ & 20.6 & 142.0 \\
$\mathrm{NOx}\left(\mu \mathrm{g} / \mathrm{m}^{3}\right)$ & $4.09(3.71)$ & 0.3 & 29.6 \\
$\mathrm{PM}_{2.5}\left(\mu \mathrm{g} / \mathrm{m}^{3}\right)$ & $13.20(5.66)$ & 0.4 & 41.3 \\
$\mathrm{TA}$ & $20.43(3.99)$ & 7.2 & 36.4 \\
\hline \#casos de internação; DP: desvio-padrão da & média; TA: \\
temperatura aparente. & &
\end{tabular}

O único poluente cuja exposição se mostrou associada às internações por DRs foram os óxidos de nitrogênio. Esta associação foi evidenciada nos lags 1 e 4, sendo que os RR para internação foram $\mathrm{RR}=1,046$ (IC95\%: 1,015-1,079) e RR=1,054 (IC95\%: 1,054-1,196) respectivamente. $\mathrm{O}$ incremento da ordem de $3 \mu \mathrm{g} / \mathrm{m}^{3}$, correspondente à diferença interquartil nas concentrações de NOx, gerou aumentos percentuais da ordem de 14,8\% (IC95\%: 4,5-25,1) para o lag 1 e 17,2 \% (IC95\%: 4,5-33,6) para o lag 4 (Tabela 2).

Neste estudo sobre os efeitos da exposição a poluentes do ar e internações por doenças respiratórias realizado em Taubaté, SP, foi possível identificar o papel dos óxidos de nitrogênio neste desfecho. É importante ressaltar que esta cidade não conta com estações medidoras da CETESB e assim foram utilizados dados estimados pelo modelo matemático CATT-BRAMS (Freitas et al., 2007). O uso dos dados estimados por esse sistema, validados por outros autores (Ignotti et al., 2010; César et al., 2013; Silva et al., 2013), permite baratear 
os custos de pesquisa e agilizar o processo de análise dos efeitos da poluição atmosférica na saúde.

Tabela 2. Valores dos riscos relativos e respectivos intervalos de confiança de 95\%, entre colchetes, para os poluentes monóxido de carbono $(\mathrm{CO})$, ozônio $\left(\mathrm{O}_{3}\right)$, óxidos de nitrogênio $\left(\mathrm{NO}_{\mathrm{x}}\right)$ e material particulado fino $\left(\mathrm{PM}_{2,5}\right)$, segundo defasagens (lag) de zero a cinco dias, Taubaté, 2011-2012.

\begin{tabular}{|c|c|c|c|c|}
\hline & $\mathrm{CO}$ & $\mathbf{O}_{3}$ & NO $_{\mathbf{x}}$ & $\mathbf{P M}_{2.5}$ \\
\hline LAG 0 & $0,998[0,995-1,001]$ & $1,001[0,994-1,007]$ & $1,018[0,979-1,059]$ & $0,985[0,965-1,005]$ \\
\hline LAG 1 & $0,999[0,996-1,001]$ & $1,001[0,994-1,008]$ & $1,046[1,015-1,079] \#$ & $0,991[0,971-1,012]$ \\
\hline LAG 2 & $1,001[0,998-1,003]$ & $0,997[0,990-1,003]$ & $1,013[0,980-1,048]$ & $1,003[0,982-1,025]$ \\
\hline LAG 3 & $0,999[0,996-1,002]$ & $1,001[0,994-1,059]$ & $1,021[0,985-1,059]$ & $1,000[0,978-1,023]$ \\
\hline LAG 4 & $0,998[0,998-1,001]$ & $0,997[0,997-1,004]$ & $1,054[1,054-1,196] \#$ & $0,984[0,984-1,007]$ \\
\hline LAG 5 & $1,000[0,997-1,002]$ & $0,995[0,989-1,002]$ & $1,023[0,990-1,058]$ & $0,998[0,975-1,020]$ \\
\hline
\end{tabular}

\# p-valor $<0,05$.

Os valores médios obtidos por modelagem matemática para as concentrações dos poluentes apresentaram-se bem abaixo dos valores encontrados por Gouveia et al. (2006) utilizando dados obtidos de 14 estações de monitoramento da CETESB na cidade de São Paulo, mas os efeitos na saúde, representados por risco de internação por doenças respiratórias, segundo exposição aos óxidos de nitrogênio, foram maiores para internação por DRs em todas as idades, que os encontrados no estudo acima citado, mesmo considerando um aumento de $10 \mu \mathrm{g} / \mathrm{m}^{3}$ nas concentrações de $\mathrm{NO}_{2}$.

Outro ponto a ser citado é que as concentrações não ultrapassaram os limites recomendados, não ocorrendo nenhum dia com qualidade do ar que não fosse considerada "boa", segundo os valores padrões estabelecidos pela CETESB (2013).

$\mathrm{O}$ fato da exposição aos outros poluentes, $\mathrm{PM}_{2.5}, \mathrm{CO}$ e $\mathrm{O}_{3}$ não se mostrarem associadas às internações, possivelmente se deva às menores concentrações destes poluentes, diferente dos resultados obtidos por Gouveia et al. (2006), pois os mesmos usaram dados reais dos poluentes atmosféricos. Mesmo o material particulado fino, que compõe até $70 \%$ do $\mathrm{PM}_{10}$, não se mostrou associado às internações como ocorreu em Piracicaba, SP, mas cuja faixa etária avaliada foi até 10 anos (César et al., 2013). No estudo realizado em Cubatão (Jasinski et al., 2011), também incluindo crianças e adolescentes, a exposição ao $\mathrm{NO}_{2}$ não se mostrou associada à internação por doenças respiratórias, mesmo com concentrações da ordem de $27 \mu \mathrm{g} / \mathrm{m}^{3}$.

Por outro lado, em Sorocaba, SP, cidade de porte médio como Taubaté, contudo com população maior e cortada por duas importantes rodovias, a exposição ao $\mathrm{NO}_{2}$ esteve associada à internação por pneumonias em crianças, sendo que as concentrações médias foram da ordem de $48 \mu \mathrm{g} / \mathrm{m}^{3}$ e eram maiores nos mesmos meses que encontradas em Taubaté (Negrisoli e Nascimento, 2013).

É difícil diferenciar e isolar os efeitos do $\mathrm{NO}_{2}$ dos efeitos dos outros poluentes, pois são concentrações fortemente correlacionadas. Neste estudo, foi considerado que as concentrações de óxidos de nitrogênio que são constituídos principalmente por $\mathrm{NO}$ e $\mathrm{NO}_{2}$, representam as concentrações de $\mathrm{NO}_{2}$ porque o $\mathrm{NO}$ rapidamente se transforma em $\mathrm{NO}_{2}$. As maiores concentrações deste poluente ocorreram nos meses de junho a setembro (gráfico não mostrado). 
Neste estudo foram incluídas as informações de pessoas internadas que eram residentes em Taubaté, podendo incluir aqueles que se internaram em cidades vizinhas. Estudos como este que utilizam dados do Sistema Único de Saúde excluem informações de internações de pacientes com planos de saúde privados ou operadoras de saúde; dessa forma, estes resultados se referem aos usuários do SUS. No entanto, a falta de informações sobre a presença de comorbidades, talvez possa representar uma limitação deste estudo, além de possíveis erros na codificação do diagnóstico. Mesmo assim, a qualidade e a confiabilidade das informações vêm sendo referendadas em outros estudos citados neste trabalho.

\section{CONCLUSÕES}

Foi possível associar a exposição ao $\mathrm{NO}_{2}$ à internação por doença respiratória utilizando dados estimados pelo modelo matemático CATT-BRAMS. Mesmo que a resolução espacial representada pelo modelo não seja a mesma da área de abrangência da população exposta aos poluentes do ar, a utilização deste modelo em cidades onde não há medidores de agências ambientais torna-se uma possibilidade, permitindo aos gestores públicos implantação de políticas de controle da emissão de poluentes com consequente diminuição de desfechos danosos à saúde.

\section{REFERÊNCIAS}

AMÂNCIO, C. T.; NASCIMENTO, L. F. C. Asma e poluentes ambientais: um estudo de séries temporais. Revista Associação Médica Brasileira, v. 58, n. 3, p. 302-307, 2012. http://dx.doi.org/10.1590/S0104-42302012000300009

ARBEX, M. A.; SANTOS, U. P.; MARTINS, L. C.; SALDIVA, P. H. N.; PEREIRA, L. A. A.; BRAGA, A. L. F. A poluição do ar e o sistema respiratório. Jornal Brasileiro de Pneumologia, v. 38, n. 5, p. 643-655, 2012. http://dx.doi.org/10.1590/S180637132012000500015

BARNETT, A. G.; TONG, S.; CLEMENTS, A. C. A. What measure of temperature is the best predictor of mortality? Environmental Research, v. 110, p. 604-611, 2010. http://dx.doi.org/10.1016/j.envres.2010.05.006

BRASIL Ministério da Saúde. Departamento de Informática do Sistema Único de Saúde. Available in: http://tabnet.datasus.gov.br/cgi/deftohtm.exe?sih/cnv/nruf.def. Access in: May 2014a.

BRASIL. Ministério da Saúde. Departamento de Informática do Sistema Único de Saúde. Available in: http://tabnet.datasus.gov.br/cgi/tabcgi.exe?sim/cnv/obt10uf.def. Access in: May 2014b.

CÉSAR, A. C. G.; NASCIMENTO, L. F. C.; CARVALHO, JR. J. A. Associação entre exposição ao material particulado e internações por doenças respiratórias em crianças. Revista de Saúde Pública, v. 47, n. 6, p. 1209-1212, 2013. http://dx.doi.org/10.1590/S0034-8910.2013047004713

COMPANHIA DE TECNOLOGIA DE SANEAMENTO AMBIENTAL - CETESB. Qualidade do ar. São Paulo, 2013. Available in: http://ar.cetesb.sp.gov.br/. Access in: Jan. 2015. 
DEPARTAMENTO NACIONAL DE TRÂNSITO - DENATRAN. Frota de veículos. Frota Nacional (outubro de 2014). Brasil, 2014. Available in: http://www. denatran.gov. br/frota2014.htm. Access in: Jan. 2015.

FREITAS, S. R.; LONGO, K. M.; DIAS, M. A. F. S.; CHATFIELD, R.; DIAS, P. L. S.; ARTAXO, P. et al. The coupled aerosol and tracer transport model to the Brazilian developments on the Regional Atmospheric Modeling System (CATTBRAMS). Part 1: model description and evaluation. Atmospheric Chemistry and Physics Discussions, v. 7, n. 3, p. 8525-8569, 2007. http://dx.doi.org/10.5194/acpd-7-8525-2007

GOUVEIA, N.; FREITAS, C. U.; MARTINS, L. C.; MARCILIO, I. O. Hospitalizações por causas respiratórias e cardiovasculares associadas à contaminação atmosférica no Município de São Paulo, Brasil. Caderno de Saúde Pública, v. 22, n. 12, p. 2669-2677, 2006. http://dx.doi.org/10.1590/S0102-311X2006001200016

HERNANDEZ, M. F. Estudio del impacto en la calidad del aire de las fuentes pontual es en la ciudad de Pinar Del Río. Revista Brasileira de Meteorologia, v. 28, n. 1, p. 1-12, 2013. http://dx.doi.org/10.1590/S0102-77862013000100001

INSTITUTO BRASILEIRO DE GEOGRAFIA E ESTATÍSTICA - IBGE. Censos Demográficos. Cidades@. Brasil, 2015. Available in: http://www.cidades.ibge.gov.br/ xtras/perfil.php?lang=\&codmun $=355410 \&$ search $=$ sao-paulo|taubate. Access in: Jan. 2015 .

IGNOTTI, E.; VALENTE, J. G.; LONGO, K. M.; FREITAS, S. R.; HACON, S. S.; ARTAXO NETTO, P. Impact on human health of particulate matter emitted from burnings in the Brazilian Amazon region. Revista de Saúde Pública, v. 44, n. 1, p. 121-130, 2010. http://dx.doi.org/10.1590/S0034-89102010000100013

JASINSKI, R.; PEREIRA, L. A. A.; BRAGA, A. L. F. Poluição atmosférica e internações hospitalares por doenças respiratórias em crianças e adolescentes em Cubatão, São Paulo, Brasil, entre 1997 e 2004. Caderno de Saúde Pública, v. 27, n. 11, p. 22422252, 2011. http://dx.doi.org/10.1590/S0102-311X2011001100017

NEGRISOLI, J.; NASCIMENTO, L. F. C. Poluentes atmosféricos e internações por pneumonia em crianças. Revista Paulista de Pediatria, v. 31, n. 4, p. 501-506, 2013. http://dx.doi.org/10.1590/S0103-05822013000400013

SILVA, A. M. C.; MATTOS, I. E.; IGNOTTI, E.; HACON, S.S. Material particulado originário de queimadas e doenças respiratórias. Revista de Saúde Pública, v. 47, n. 2 , p. 345-352, 2013. http://dx.doi.org/10.1590/S0034-8910.2013047004410 